\title{
Le Service de la recherche de la SNCF vu par Roger Guibert
}

Roger Guibert

\section{OpenEdition}

\section{Journals}

Édition électronique

URL : https://journals.openedition.org/rhcf/814

DOI : $10.4000 /$ rhcf.814

\section{Éditeur}

Rails \& histoire

Édition imprimée

Date de publication : 1 juin 2008

Pagination : $33-43$

ISSN : 0996-9403

Référence électronique

Roger Guibert, "Le Service de la recherche de la SNCF vu par Roger Guibert », Revue d'histoire des chemins de fer [En ligne], 39 | 2008, mis en ligne le 01 juin 2011, consulté le 22 avril 2022. URL : http:// journals.openedition.org/rhcf/814 ; DOI : https://doi.org/10.4000/rhcf.814 


\section{Le Service de la recherche de la SNCF vu par Roger Guibert \\ François Caron a choisi de publier le document suivant, extrait des Archives historiques de la SNCF (CAH, 505 LM), qui rejoint des travaux antérieurs de l'AHICF sur l'histoire des grandes vitesses en France et ouvre un cours de réflexion sur les chemins de l'innovation ferroviaire.}

Le 15 décembre 1971 Roger Guibert présente au conseil d'administration de la SNCF les travaux du Service de la recherche depuis 1966. Parmi les quarante-deux rapports rédigés et les vingt projets entrepris, il sélectionne "les plus caractéristiques par leurs aspects pluridisciplinaires et prospectifs». Ces deux mots rendent clairement compte de l'esprit qui a inspiré les fondateurs et les animateurs du service. La pluridisciplinarité se manifeste à la fois par la coopération que le Service de la recherche a su instaurer entre les différents services de l'entreprise au sein des "groupes de travail 》 que par l'ouverture vers des organismes de recherche, des bureaux d'études et des entreprises extérieurs. Ainsi est institué un "dialogue permanent entre les chercheurs, les bureaux d'études et les utilisateurs ». La nature prospective s'exprime par la volonté d'ouvrir de nowveaux domaines de recherche tels que "les recherches économiques de caractère scientifique » mais aussi par le désir de mettre au service de "l'outil ferroviaire existant [...] l'utilisation des techniques et des méthodes les plus avancées ». La SNCF entre ainsi dans l'ère des hautes technologies et de la prospective à long terme qui n'a jamais cessé, depuis lors, d'influencer son mode de gestion. Ce texte montre enfin que la création du Service de la recherche ne constitue pas une rupturet. On y perçoit bien des échos de l'enthousiasme des premiers jours que Guibert a vécu, ou de l'esprit modernisateur des disciples de Dautry, de Lonis Armand et de quelques autres...

François Caron

1- Voir « Aux origines des très grandes vitesses ferroviaires en France : histoire d'une décision, genèse d'une innovation ", Actes de la sixième journée scientifique de l'AHICF (31 mars 1994), Revue d'bistoire des chemins de fer $\mathrm{n}^{\circ} 12-13$ (printemps-automne 1995), qui suivait une enquête orale sur le même sujet commandée par l'AHICF au CNRS, bientôt en ligne sur le site www.memoire-orale.org. 


\section{ANNEXE AU PROCÈS-VERBAL DE LA SÉANCE DU CONSEIL D'ADMINISTRATION DU 15 DÉCEMBRE 1971 EXPOSÉ de M. GUIBERT, Directeur Général sur l'activité du Service de la Recherche de 1966 à 1971}

Le Service de la Recherche a maintenant 5 ans d'existence. C'est, en effet, le 27 juillet 1966 que j'avais exposé, devant le Conseil, les raisons pour lesquelles, bien que la S.N.C.F. menât déjà une politique active de recherches ainsi qu'en témoignaient les résultats obtenus dans de nombreux domaines, l'organisation existante ne paraissait plus suffisante si l'on entendait garantir l'avenir du chemin de fer au milieu d'autres modes de transport de plus en plus perfectionnés. En fait, nos recherches n'étaient complètes qu'en ce qui concerne le perfectionnement de l'outil actuel, et leur degré d'approfondissement variait en fonction inverse du nombre de Services appelés à y participer. Les recherches prospectives n'étaient encore que fragmentaires, ne couvrant pas systématiquement l'ensemble des problèmes, et des lacunes subsistaient dans le domaine des recherches économiques de caractère scientifique.

Dans ces conditions, un groupe de travail avait été chargé d'étudier une nouvelle organisation de nos Services de recherche. Il préconisa la création d'un organisme nouveau qui, tout en laissant à chaque Direction sa part de recherches intégrée - celle qui est liée aux études courantes de ces Directions -, centraliserait tout ce qui peut avoir un caractère prospectif et pluridisciplinaire, c'est-à-dire intéressant plusieurs spécialités ferroviaires : mouvement, activité commerciale ou action technique.

\section{L'ORGANISATION ET LES} MÉTHODES DE TRAVAIL du nouveau Service qui, à la fin de l'année 1966, fut chargé de cette mission, devaient évidemment tenir compte des particularités de cette dernière. Celles qui ont été alors adoptées peuvent, au bout de 5 ans, être considérées comme ayant fait leurs preuves.

Je rappelle d'abord que le Service de la Recherche dispose actuellement de quelque 130 agents qui, abstraction faite des tâches afférentes à l'administration et à la documentation, sont groupés par discipline dans cinq Départements : "Technique", "Scientifique", "Exploitation", "Cybernétique" et "Économie". Il n'est pas envisagé de dépasser cet effectif qui est notablement inférieur à celui de 200 à 300 agents indiqué en 1966, mais qui parait bien correspondre aux besoins actuellement prévisibles. 
Ce personnel du Service de la Recherche a été recruté dans les Régions et dans les autres Services de la Direction Générale, avec le souci, toutefois, de ne pas trop dégarnir les Divisions d'études des Directions techniques. En principe, les agents ainsi affectés au Service de la Recherche, où ils apportent l'expérience pratique de leur spécialité, retournent, après quelques années, dans leur service d'origine, enrichis d'une formation complémentaire particulièrement intéressante sur les problèmes fondamentaux du chemin de fer. Je signalerai notamment que le Service de la Recherche a fourni, pour les nouvelles structures, deux Directeurs de Région et un Directeur Adjoint.

Les chercheurs de chaque Département s'occupent, bien entendu, en priorité, des études et projets qui intéressent directement le Chemin de fer et que je vais tout à l'heure évoquer dans leurs lignes essentielles, mais ils consacrent également une partie notable de leur activité et de leur temps à des travaux qui concernent leur discipline propre, notamment pour tout ce qui touche les approches scientifiques et méthodologiques nouvelles. À cet effet, ils doivent faire largement appel à des organismes extérieurs et même à des recrutements d'experts contractuels dans les spécialités cybernétiques, économiques et mathématiques. Cette ouverture sur l'extérieur a en outre l'avantage de faire mieux ressentir la présence de la S.N.C.F. et l'importance des problèmes ferroviaires dans les milieux scientifiques ou économiques concernés par l'avenir des transports, qu'il s'agisse d'organismes publics, d'instituts de recherche publics ou privés, d'universités ou de sociétés d'études.

Parmi ces organismes avec lesquels nous sommes en rapport, je citerai :

. sur le plan national:

- l'Institut de Recherche des Transports (I.R.T.),

- la Direction de la Prévision au Ministère de l'Économie et des Finances, - le Service des Affaires Économiques Internationales (mission de la recherche), au Ministère des Transports, - la Délégation à l'Aménagement du Territoire et à l'Action Régionale (D.A.T.A.R.),

- la Délégation Générale à la Recherche Scientifique et Technique (D.G.R.S.T.),

- le Centre National de la Recherche Scientifique (C.N.R.S.),

- le Centre National d'Etudes des Télécommunications (C.N.E.T.),

- les grandes écoles d'ingénieurs ;

. sur le plan international:

- le "Department of transportation" (DOT) au Ministère des Transports des États-Unis d'Amérique,

- l'Institut des Transports Aériens (I.T.A.),

- les Services de recherche et de planification prospectives des principaux Réseaux de chemins de fer européens et, notamment, la Commission de Recherche Prospective de l'UIC. 
En fait, ces contacts extérieurs se concrétisent souvent par des contrats de recherche dans des domaines où la S.N.C.F. ne dispose pas de moyens ou d'expérience suffisants. 37 contrats de cette nature ont été conclus depuis la création du Service de la Recherche, pour un montant global de 5,3 M.

J'en citerai quelques-uns qui me paraissent propres à donner une idée du genre de recherches que nous demandons à des organismes extérieurs.

Nous avons ainsi passé, à propos du projet d'automatisation de la circulation (dit projet A 31), un contrat :

- avec la C.I.I. (Compagnie Internationale pour l'Informatique), pour l'étude et la réalisation d'un système de régulation automatique,

- avec IBM pour l'étude d'un système central de fluidification par ordinateur digital,

- avec la SNECMA pour la fourniture d'une "mémoire active" de laboratoire, dont j'aurai l'occasion de dire un mot tout à l'heure,

- avec la Compagnie de Signaux et d'Entreprises Électriques (C.S.E.E.), pour l'étude et la réalisation d'un prototype de simulateur de réseau,

- avec la Compagnie Électro-Mécanique, Faiveley-Secheron, en vue de la fourniture d'équipements prototypes pour la marche programmée des automotrices "sud-ouest".

Pour le projet relatif aux infrastructures nouvelles (projet C 03), le C.N.R.S. et la S.E.S.S.I.A. (Société d'Études et de Constructions, de Souffleries, de Simulateurs et d'Ins- trumentation Aérodynamique) se sont vu confier des études aérodynamiques de base sur les effets de la circulation des trains à très grande vitesse.

Quant au projet concernant la "fiabilité" (projet A 91), il a entraîné la conclusion de trois contrats avec MATRA pour les études de fiabilité de divers équipements et composants et d'un contrat avec la C.S.E.E. pour la fiabilité des systèmes de signalisation susceptibles d'être retenus dans le cadre du projet Paris-Lyon.

En ce qui concerne l'économie et 1'économétrie, ont été passés un contrat avec MATRA pour l'étude des interactions horaires-demande, un contrat avec l'IRIEC (Institut de Recherche et d'Informatique de l'Économie) pour la détermination de l'impact économique d'une ligne nouvelle sur les entreprises régionales et deux contrats avec l'I.T.A. pour des études documentaires sur le transport aérien.

Enfin, en ce qui concerne les technologies nouvelles, ont été conclus un contrat avec Alsthom pour l'étude d'une pile à combustible et un autre avec l'Université de Grenoble pour l'étude d'une suspension magnétique.

Mais l'originalité essentielle de la méthode de travail suivie par le Service de la Recherche apparaît nettement dans l'étude des "projets" de recherche pluridisciplinaires. La procédure est la suivante : une fois inscrit au programme de recherche, chaque "projet" est confié à une 
équipe conduite par un haut fonctionnaire de la S.N.C.F. qualifié "directeur de projet". Chaque équipe comporte, non seulement des membres du Service de la Recherche, mais également des spécialistes des diverses Directions centrales (notamment des Divisions d'études de ces Directions) et même, dans certains cas, des Régions. Les directeurs de "projets" appartiennent généralement au Service de la Recherche, mais ils sont amenés à créer des équipes spécialisées dont les animateurs peuvent être choisis en dehors de ce Service de la Recherche et entre lesquelles les différents travaux d'un même projet sont répartis. À titre d'exemple, le projet $\mathrm{C} 03$ - qui, comme nous l'avons vu ci-dessus, se rapporte aux possibilités ferroviaires sur infrastructures nouvelles et, plus spécialement au projet de desserte de Paris-Lyon à très grande vitesse - a comporté 4 sous-projets et a nécessité la constitution de 15 équipes associant plus de 100 personnes, tant du Service de la Recherche que des Directions centrales et des Régions de la S.N.C.F.

Ainsi, notre organisation a pour but d'intégrer avec une souplesse suffisante tous les moyens d'étude de la S.N.C.F. en instituant un dialogue permanent entre les chercheurs, les bureaux d'études et les services utilisateurs.

Le programme de travail du Service de la Recherche est fixé, sur le plan national, par la Commission de la Recherche qui est présidée par moi-même ou par l'un de mes Adjoints, et à laquelle assistent, outre le Chef du Service en cause, les Directeurs des principaux Services centraux. C'est elle qui, dès 1967, a arrêté le premier programme de travail du Service de la Recherche, programme remanié et complété pendant les exercices suivants.

Au total, depuis la création du Service de la Recherche, fin 1966, la Commission de la Recherche a tenu 39 séances (en moyenne 8 par an), elle a examiné 42 rapports présentant les conclusions d'études relatives aux projets de recherche, et elle a évidemment décidé des suites à donner à ces rapports.

C'est également cette Commission qui, chaque année, établit le projet de budget de la Recherche, lequel intéresse à la fois le Service de la Recherche et les Services d'études des Directions techniques.

\section{LES TRAVAUX DU SERVICE} DE LA RECHERCHE ont été d'abord organisés autour de trois thèmes fondamentaux :

$1^{\circ}$ ) Quel résultat optimal peut-on tirer de l'outil ferroviaire existant grâce à l'utilisation des techniques et des méthodes les plus avancées?

$2^{\circ}$ ) Quelles sont, dans une perspective à long terme, les caractéristiques économiques et les possibilités du transport ferroviaire?

$3^{\circ}$ ) Convient-il, pour répondre à la demande future, d'avoir recours à 
des nouvelles techniques de transport terrestre guidé, même non ferroviaires au sens strict de l'expression?

Un 4ème thème est venu ultérieurement préciser et compléter les précédents : quelles perspectives l'horizon 1985 offre-t-il à la S.N.C.F. ?

Je ne puis, bien entendu, entrer dans le détail des 42 rapports et des 20 "projets" encore en cours d'étude. J'évoquerai néanmoins les plus caractéristiques par leurs aspects pluridisciplinaire et prospectif.

- Le projet A 11, qui concerne l'acheminement des marchandises, l'un des problèmes fondamentaux de l'exploitation ferroviaire, comporte deux sous-projets. L'un, relatif à l'amélioration rapide du Régime Ordinaire, a donné lieu, depuis mars 1968, à plusieurs conclusions suivies d'exécution. L'autre est afférent à la possibilité de créer un Régime Unique se substituant aux deux Régimes actuels (Ordinaire et Accéléré); il s'agit là d'une question extrêmement importante et très délicate; si le stade du défrichement a été dépassé, celui du difficile inventaire des avantages et des inconvénients que présenterait ce Régime Unique ne l'est pas et des études complémentaires ont dû être prescrites.

- Le projet A 22 a pour objet la définition du matériel roulant pour les trains de voyageurs. Il inclut, entre autres, le sous-projet "rames à turbine à gaz", dont les conclusions ont débouché sur la mise en service des ETG sur Paris-Cherbourg, puis sur la commande des RTG pour la desserte de lignes transversales, notamment. Dans le cadre de ce même projet, un nouveau sous-projet a été retenu : il tend à définir, pour le long terme, les caractéristiques d'un matériel électrique adapté à une desserte voyageurs de jour à moyenne distance, dans des conditions comparables à celles que satisfait actuellement le matériel à turbine.

- Le projet A 31 (Automatisation de la circulation) relève plus que d'autres, peut-être, de l'anticipation.

Son premier objectif est d'augmenter le débit des trains sur les infrastructures existantes.

Il a comporté, en particulier, un sous-projet "Paris-Juvisy", grâce auquel ont été définis les moyens qui, en accroissant les possibilités de trafic de $30 \%$ par rapport à 1967, permettraient de différer la construction d'une 5ème voie. Ce sous-projet a donné lieu ou va donner lieu à divers travaux exécutés par étapes; une première opération consiste à remanier le block automatique de ce secteur et à établir un PRS à hauteur de Choisy-le-Roi, travaux qui sont inscrits au budget de 1972. On remarquera, incidemment, qu'une augmentation de quelque $30 \%$ du débit de la ligne Paris-Juvisy correspondrait à une utilisation maximale des possibilités du tronçon Paris-Austerlitz - Paris-Quai d'Orsay. 
Un autre sous-projet d'automatisation vise à augmenter dans toute la mesure du possible le débit du "triangle" de Gagny où passent chaque jour quelque 300 trains ; différents systèmes automatiques ont été définis pour assurer une "fluidification" optimale des circulations et ces systèmes font l'objet de diverses expérimentations.

Dans cet ordre d'idées, je ferai une courte digression pour signaler l'intérêt d'un simulateur de type original qualifié "mémoire active" et inventé par un chercheur étranger à la Société Nationale, le Docteur Sauvan, aux travaux duquel, néanmoins, nous avons collaboré si étroitement que la S.N.C.F. vient de recevoir pour la mise au point de cette mémoire active, conjointement avec cet inventeur, le prix "Apollo de l'Innovation". Pour maints problèmes que nous avons à résoudre, le recours à l'ordinateur de type classique est théoriquement possible grâce à l'existence des méthodes séquentielles, mais celles-ci, pour d'autres problèmes, conduiraient à envisager un nombre de combinaisons si élevé (s'exprimant par millions ou centaines de millions) que les temps de calcul deviendraient prohibitifs. Le simulateur de "mémoire active" utilise des méthodes plus intuitives, moins complexes, moins rationnelles aussi, peut-être, mais qui aboutissent à une approximation très satisfaisante. Son mécanisme, plus proche, paraitt-il, de celui du cerveau humain, doit permettre, précisément, de résoudre, dans des limites de temps raisonnables, les problèmes qui nous intéressent dans le domaine de la régulation du trafic ferroviaire ou de l'affectation des moyens, qu'il s'agisse de machines, d'hommes ou d'éléments d'infrastructures, voies ou quais par exemple.

Toujours en ce qui concerne l'automatisation de la circulation, une étape de recherche à plus long terme va être entreprise pour définir un système plus achevé d'automatisation de la circulation. Elle pourrait néanmoins comporter des applications à échéance relativement proche, allant jusqu'à l'automatisation complète d'une ligne.

- Le projet A 91 n'a été définitivement arrêté qu'en janvier 1970 et touche un champ d'étude tout à fait nouveau pour la S.N.C.F. Il a pour but d'évaluer l'intérêt des méthodes de calcul de fiabilité dans les différents secteurs d'activité de la S.N.C.F. Il existe maintenant, en effet, dans les domaines techniques, des méthodes qui permettent d'établir des prévisions de défaillance d'un matériel donné. Elles consistent à rassembler des moyens mathématiques fournissant la possibilité de faire des analyses statistiques sur le comportement des composants et des systèmes, et de prévoir les risques de défaillance.

Lorsqu'il s'agit d'un matériel utilisant des composants connus, dans des environnements toujours semblables, 
l'analyse statistique de son comportement peut être effectuée a priori, en partant de données expérimentales qui ont été antérieurement collectées sur les composants eux-mêmes ; c'est le cas des appareils électroniques, pour lesquels il est possible de faire des calculs de fiabilité prévisionnelle.

Lorsqu'il s'agit d'un matériel spécifique, la fiabilité ne peut être calculée qu'à partir des données expérimentales recueillies sur ce matériel, à moins qu'il [n'] existe déjà un matériel similaire en service. Le calcul permet de quantifier les risques de défaillance de ce matériel au cours de sa mission, de préciser le degré de confiance à accorder à cette quantification et, corrélativement, d'orienter les services d'étude et de contrôle.

Dans le cadre de ce projet, nous cherchons à évaluer l'intérêt pratique de ces méthodes de fiabilité pour le chemin de fer et également à former des spécialistes. Les techniques de fiabilité étant surtout développées dans le domaine de l'aérospatiale, nous avons exécuté des études conjointement avec la Société MATRA pour bénéficier de son expérience, études qui ont confirmé la valeur de ces méthodes.

Leur importance dans le cas du projet $\mathrm{C} 03$ de ligne à très grande vitesse mérite d'être soulignée. L'utilisation intensive du matériel qui est à prévoir impose, en effet, un effort particulier pour réduire les risques de panne et de défaillance. Les études portent plus spécialement sur la fiabilité de certains composants de l'équipement des rames $\mathrm{C} 03$ et sur celle des systèmes de signalisation.

Le rôle du Service de la Recherche est, bien entendu, d'assumer la direction technique de ces études, de les coordonner et de contrôler la validité des méthodes utilisées tout en assurant aux agents qui y participent le complément de formation qui peut leur être nécessaire.

- Les projets B 11 (Bilan économique de la vitesse) et B 12 (Détermination des frais d'infrastructures imputables aux différentes catégories de prestations ferroviaires) doivent contribuer à une meilleure application de la doctrine économique en matière de transport ferroviaire.

- Les projets B 21 (Analyse de la demande "voyageurs") et B 22 (Analyse de la demande "marchandises") sont des recherches théoriques sur les facteurs d'offre susceptibles de réagir sur la demande. Méritent notamment d'être signalées les études approfondies d'ores et déjà réalisées concernant le prix auquel l'usager est disposé à payer un gain de temps et l'influence de la fréquence des trains sur le volume du trafic voyageurs; leurs conclusions ont d'ailleurs été exploitées pour le projet C 03 . 
- Le projet C 02 intéresse 1'aérotrain. Nous avons publié en 1968 un rapport sur la situation des recherches et expérimentations à cette époque. Depuis lors, le Service de la Recherche continue de diriger toutes les études qui concernent le coussin d'air, question sur l'importance et l'actualité de laquelle il est inutile d'insister.

- Le projet $\mathrm{C} 03$ a déjà été souvent évoqué ci-dessus.

Son principal sous-projet, celui de la ligne à très grande vitesse ParisLyon, est bien connu du Conseil devant lequel M. le Président a fait le point de la question le 31 mars dernier.

Mais le projet C 03 comporte d'autres sous-projets. Les possibilités d'exploitation ferroviaire sur infrastructures nouvelles sont étudiées au plan international, en fonction d'échéances relativement lointaines certes, mais dont il importe de se préoccuper dès à présent. Il s'agit, en particulier, des perspectives nouvelles qu'ouvrirait le percement du tunnel sous la Manche et nous étudions avec les Réseaux concernés l'intérêt de relations à très grande vitesse sur le triangle Londres-Paris-Bruxelles et audelà de ce triangle, vers l'Allemagne et la Hollande.

- Les études qui se rattachent au projet D 01 (études "transports" du Plan ; la S.N.C.F. en 1985) ont été entreprises en 1968 en liaison avec le Comité directeur des "Études
Transports 1985", chargé par le Commissariat du Plan de dégager les principales caractéristiques que pourrait présenter le secteur des transports eu 1985. L'intérêt de cette recherche est éventuellement de permettre la définition d'un plan directeur à long terme du chemin de fer. Malgré les aléas que comporte une telle projection dans un avenir assez lointain, on peut tout de même en tirer bénéfice pour l'orientation de nos plans d'entreprise à plus court terme.

- Enfin, j'évoquerai les projets d'études régionales qui sont également élaborés dans la perspective de "l'horizon 1985" et qui ont pour objet d'apprécier l'évolution de l'offre et de la demande de transport le long de certains axes ferroviaires ou dans certaines régions où peuvent se poser, à relativement brève échéance, des problèmes de capacité. Quand on examine la prospective du chemin de fer pour les dernières années du siècle, on est conduit, même en recourant à des évaluations très prudentes, à multiplier le trafic par 2,5, ce qui soulève des questions de capacité assez importantes dont il faut se préoccuper longtemps à l'avance. Ces recherches de capacité sont actuellement concentrées sur l'axe ParisDijon-Lyon-Méditerranée, les vallées alpines - où se multiplient les dessertes des stations de sports d'hiver -, la Région Parisienne et la Basse-Seine. 
Toujours dans le domaine de la CYBERNÉTIQUE, le Service de la Recherche a montré ce que pouvait être l'apport des méthodes de simulation pour la résolution de problèmes ferroviaires, notamment lorsqu'il s'agit de phénomènes trop complexes pour être décrits par des modèles analytiques. Ces méthodes semblent devoir être appelées à un large développement, en matière économique et opérationnelle, voire pour certains problèmes touchant à la gestion du personnel.

D'autres recherches cybernétiques concernent l'élaboration de modèles analytiques appliqués au débit des lignes, les études d'implantation et de gestion automatique des installations permanentes de contresens, les applications de la Mémoire Active, la transmission d'informations numériques au moyen d'une liaison radio préexistante, etc.

Dans le domaine TECHNIQUE, les travaux dont le Service de la Recherche prend l'initiative sont essentiellement de nature théorique. Ils ont leur origine dans l'existence de méthodes nouvelles tant pour la formulation mathématique de certains problèmes complexes (problèmes mécaniques, notamment) que pour les résolutions de systèmes d'équations auxquels cette formulation conduit presque toujours. Ces méthodes nouvelles, rendues possibles par l'utilisation des calculatrices électroniques, nécessitent, pour leur mise en œuvre, des spécialistes joignant une compétence combinée de mécanicien, de mathématicien et d'informaticien. C'est ainsi, par exemple, que la connaissance de la programmation linéaire pour la résolution de problèmes économiques, a suggéré l'application de cette programmation à des problèmes techniques.

Le Service de la Recherche a, sous cet angle, étudié, en particulier, l'optimisation de la rectification des courbes de voie, la stabilité transversale d'un véhicule circulant sur une voie présentant des défauts, l'aérodynamisme instationnaire, l'utilisation des freins à courant de Foucault, et apporté, pour diverses autres études, une aide de nature informatique et scientifique aux Directions fonctionnelles.

Indépendamment des études économiques nécessitées par les divers projets, les recherches économiques auxquelles se livre le Service précité se situent pour la plupart dans le cadre d'une collaboration avec la Direction Commerciale et la Direction des Études Générales. Mentionnons, à cet égard, l'élaboration et l'exploitation de modèles de prévisions de la demande voyageurs (modèles prix-temps, modèles à coût généralisé, modèles plurimodaux), la mise au point de méthodes d'analyse multidimensionnelle de fichiers de données (qui ont d'ailleurs une portée générale dépassant les seuls besoins commerciaux), certains travaux concernant les méthodologies de choix des investissements et les conséquences de la crise de l'énergie. 
La construction de modèles analytiques ou de simulation s'est également introduite dans les RECHERCHES "TRANSPORT" nécessitées par l'analyse des perspectives à long terme du chemin de fer : modèle analytique prospectif du plan de transport, modèle simplifié de circulation à coût minimal sur un réseau, études de capacité d'un réseau...

Indépendamment de ses recherches dans les divers domaines spécifiques intéressant toutes les disciplines du chemin de fer, le Service de la Recherche participe à de très nombreux travaux effectués à la demande d'un ORGANISME EXTÉRIEUR NATIONAL (missions ministérielles concernant l'apport des techniques nouvelles au transport terrestre guidé ou l'élaboration des stratégies pour la mise en place des transports interrégionaux de voyageurs, Club des Transports Rapides, Commissariat du Plan, expertises pour la DGRST...) OU INTERNATIONAL : U.I.C. (Commission "Recherche Prospective"), O.R.E., Groupe des Neuf, Comité Commun du Tunnel, Commission Économique et Sociale pour l'Asie et le Pacifique (CESAP), Coopération franco-soviétique et avec le Ministère des Transports des U.S.A.
La contribution à ces études représente une charge importante. On doit cependant observer que la plupart des travaux correspondants ont des retombées positives sur ceux du Service car les objectifs de recherche sont de même nature. Ils permettent, de plus, d'avoir accès aux activités des organismes de recherche extérieurs.

Enfin, le Service de la Recherche consacre une part non négligeable de ses activités à la fonction "enseignement" dans plusieurs Grandes Écoles ou Organismes universitaires.

Voici, très rapidement survolées, ce qu'ont été les principales activités du Service de la Recherche au cours des derniers exercices, activités - on le voit - très variées qui vont de la conduite de grands projets pluridisciplinaires à des études assez fondamentales effectuées pour tels ou tels domaines spécifiques du chemin de fer où le Service de la Recherche joue en quelque sorte un rôle de prospection. 
